\title{
Research on Trading Strategies of Continuous Double Auction Based on Limited Resources
}

\author{
Yuan Shao, Meng-ya Zhou, Ya-juan Wang* \\ Center of Service Science and Engineering, School of Management \\ Wuhan University of Science and Technology, Wuhan, Hubei \\ shaoyuan@wust.edu.cn,wangyajuan@wust.edu.cn
}

\begin{abstract}
In the double auction market with limited resources, to explore how to obtain greater market total profit, and make the allocation of market resources more effective. We have developed a global model to calculate the total market profit of the multi-stage market trading strategy and the price priority strategy. Then we calculated the total market profit of the two trading strategies with the calculation example. Finally, we use excel software to simulate the five network resource allocation systems. After analyzing and comparing the experimental results of the trading strategies, we conclude that multi-stage market trading strategy exceeds the price priority strategies for the total market profit. These trading strategies can be applied to the transportation service market.
\end{abstract}

Keywords-Double auction; The multi-stage market trading strategy; The price priority strategy; Total market profit

\section{INTRODUCTION}

As computers and communication technologies developed rapidly, traditional centralized resource allocation methods have gradually been replaced by virtual transaction in the network environment. In the large-scale and open network environment, scholars have proposed a Continuous Double Auction (CDA) mechanism to effectively allocate resources. Continuous Double Auction (CDA) is a decentralized market mechanism for effective resource allocation. There are multiple sellers and buyers in the market. Sellers and buyers can submit quotes at any time, As long as the buy's bid is not lower than the seller's asking price, transaction can occur immediately [1]. Under the network resource allocation framework based on continuous double auctions, the resource allocation system consists of resource agents (providing resources), task agents (demand resources) and auctioneers (auction centers) [2]. The auctioneer combines multiple buyers and multiple sellers on the same platform to conduct transactions. Each buyer and seller is faced with multiple trading objects, but one buyer only deals with one seller and one seller only deals with one buyer eventually. Shengfeng Chen and Chengiian Wei [3] proposed in the GD2 strategy that a seller can conclude deals with multiple buyers.

Aiming to get more effective distribution of market resources, and obtain greater total market profits, the article discussed two kinds of many-to-many trading strategies that each seller can reach deals with multiple buyers, and each buyer also can enter into deals with multiple sellers. The first trading strategy in this paper is the price priority strategy. Under the premise that the buyer's bid is higher than the seller's asking price, the buyer trades with the seller who has the lowest asking price, and the seller trades with the buyer who has the highest bid. The second trading strategy is the multi-stage market trading mechanism, the first stage of trading is transaction volume (transaction price multiplied by the number of transactions) priority strategy, that is to say the first transaction occurs between the buyer and the seller that have maximum trading volume. When the first stage of trading ends, the price priority strategy is considered to complete the remaining buyer and seller's transactions. Finally, this paper compares two kinds of trading strategies through experimental data to conclude that the multi-stage market trading mechanism allows resources distribution to be more effectively, and to obtain greater total market profit.

\section{CDA Market Trading RULES}

The trading process of the CDA market under the feature of limited agent resources: sellers and buyers successively submit asking price information and bid information, and arrange these information in the price information table. The specific agreement of the market continuous trading is as follows:

- $\quad$ The seller continuously submits asking price information ask (qi, pi, i), indicating that the seller $\mathrm{i}$ is willing to provide up to qi units of resources at the unit price pi.

- $\quad$ The buyer continuously submits the bid information bid (qj, pj, j), indicating that the buyer $\mathrm{j}$ is willing to purchase the qj unit resources at the pj unit price.

- $\quad$ The seller's asking price information and the buyer's bid information are respectively arranged in the price list information table. The sellers are sorted in ascending order according to the asking price pi. If the asking price is the same, they are sorted in chronological order. The buyers sort in descending order of the bid price pj, and if the bid price is the same, Arranged in chronological order.

- Market trading rules: When the market submits a new quotation information (buyer's bid price information or seller's ask price information), the auction center (information service agent) tries to update the market, once the prices match each other transaction can occur. To update the price information table. When the resource transaction price is set to the equilibrium price (the average value of highest asking price the lowest bid that can be reached deals), resources distribution is the most efficient in the market ${ }^{[4]}$. 
For the network environment with limited agent resources, Shengfeng Chen [5] proposed a ZI2 strategy: buyers bid on pj from 0 to blj subject to uniform distribution. The seller $i$ predicts the expected trading volume sqi from 0 to cpi, uses the fixed start cost fi and the unit cost ui to calculate the floor price sli (the lowest price the seller is willing to accept), and the asking price pi goes from sli to Oask (the market permits the highest asking price of sellers) subject to uniform distribution. The ZI2 strategy is as follows:

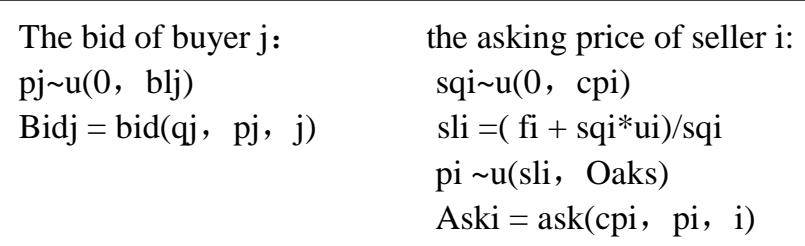

\section{TRADING STRATEGY MECHANISM.}

A. Strategy one: Price priority strategy.

- $\quad$ Arrange the seller's asking price in ascending order and place the buyer's bid in descending order.

- The buyer $\mathrm{j}$ with the highest bid and the seller with the lowest bid are traded under the premise that buyer j's bid is higher than the seller i's asking price.

- If the demand qj of the buyer $\mathrm{j}$ is greater than the offer qi of the seller $i$, the seller $i$ finishes trading, the buyer $\mathrm{j}$ still has remaining (qj-qi), then the buyer $\mathrm{j}$ continues to trade with the remaining seller with the lowest asking price in the transaction yet not to be sold, until buyer $\mathrm{j}$ finishes trading ; if the demand qj of buyer $\mathrm{j}$ is less than the offer qi of the seller $i$, buyer $j$ finishes trading, seller $i$ still has remaining (qi-qj), then the seller $i$ continues to trade with the remaining buyer $\mathrm{j}$ with the highest bid in the transaction yet not to be sold, until the seller $\mathrm{i}$ finishes trading.

- $\quad$ Trading in accordance with rule $\mathrm{c}$, when the buyer's bid price is lower than the selling asking price, the transaction ends.

\section{B. Strategy two: Multi-stage market trading mechanism}

- $\quad$ Arrange the seller's asking price in ascending order and place the buyer's bid price in descending order.

- Under the premise that the bid price of the buyer is higher than the asking price of the seller, calculate the transaction amount (the transaction price multiplied by the transaction amount) of the buyer $\mathrm{j}$ and all the sellers, and select the maximal transaction amount Wij from all the transaction amount, then seller $i$ and buyer $j$ conduct a transaction. And remove the seller $\mathrm{i}$ and the buyer $\mathrm{j}$ from the ranking.

- Continue to deal with untraded sellers and buyers in accordance with Rule b. When the buyer's bid is less than the asking price, the first round of trading ends.

- Calculate the remaining volume of all buyers and sellers after the first round of transactions, and then trade the remaining volume of all buyers and sellers according to the price priority strategy. When the buyer's bid is less than the asking price, the transaction ends.

\section{The CAlculation Model of Profit Distribution}

In the CDA market, a price-priority trading strategy and a multi-stage market trading strategy are adopted. The calculation method of the total profit actually obtained of two kind of trading strategies is the same, the buyer's total profit $\mathrm{Pb}$ the total sales profit Ps, and the actual total market profits Ptotal is

$$
\begin{aligned}
& p_{b}=\sum_{j \in I_{b}, i \in I_{s}} q_{i j}\left(v_{j}-p_{i j}\right) \\
& p_{s}=\sum_{i \in I_{s}, j \in I_{b}} q_{i j}\left(p_{i j}-s l_{i}\right) \\
& \sum_{i \in I_{s}} q_{i j} \geq d_{j} \\
& \sum_{j \in I_{b}} q_{i j} \leq c p_{i} \\
& p=p_{b}+p_{s}
\end{aligned}
$$

$I_{b}$ is a set of buyers who have conducted a transaction, $I_{s}$ is a set of sellers who have conducted a transaction, $q_{i j}$ is the of transactions volume between the seller $i$ and the buyer $j$, $v_{j}$ is the valuation of the buyer $\mathrm{j}, p_{i j}$ is the trade price of the seller $\mathrm{i}$ and the buyer $\mathrm{j}, s l_{i}$ is the bottom price of the seller $\mathrm{i}$, $d_{j}$ is the demand of the buyer $\mathrm{j}, c p_{i}$ is the provide capacity of the seller $\mathrm{i}$.

\section{EXAMPLE}

Assume that there are four sellers and four buyers in the system for resource allocation. Their basic information is as follows table 1. (The market stipulates that the highest asking price of the seller is 22 , and the lowest bid of the buyer is 10)

TABLE I BASIC INFORMATION OF BUYERS AND SELLERS.

\begin{tabular}{c|c|c|c|c}
\hline fi & ui & cpi & dj & vj \\
\hline 41 & 2.18 & 135 & 102 & 27 \\
\hline 40 & 2.70 & 95 & 98 & 25 \\
\hline 20 & 2.85 & 90 & 80 & 21 \\
\hline 43 & 3.20 & 82 & 85 & 27 \\
\hline 35 & 3.50 & 73 & 79 & 24 \\
\hline
\end{tabular}

For sellers: First, suppose that the seller in the first row has an expected transaction volume sqi 120 , and then calculates the base price of the seller in the first row.

$$
s l_{i}=\frac{f_{i}+u_{i}{ }^{*} s q_{i}}{s q_{i}}=\frac{41+2.18^{* 120}}{120}=2.52
$$

Therefore the asking price range of the seller in the first row is $(2.52,22)$, assuming that the asking price of the seller in the first row is 8 . 
For buyers: The first row of buyers has a range of bid price $(10,27)$, assuming that the buyer in the first row bids on 20.

TABLE II QUOTATION INFORMATION OF BUYERS AND SELLERS

\begin{tabular}{c|c|c|c|c|c}
\hline sqi & sli & cpi & Pi & pj & dj \\
\hline 120 & 2.52 & 135 & 8 & 20 & 102 \\
\hline 90 & 3.10 & 95 & 12 & 16 & 98 \\
\hline 85 & 3.09 & 90 & 16 & 13 & 80 \\
\hline 75 & 3.77 & 82 & 10 & 14 & 85 \\
\hline 68 & 4.01 & 73 & 14 & 18 & 79
\end{tabular}

Strategy one: The trading process and result of the price priority strategy is as following.

TABLE III TRADING PROCESS OF THE PRICE PRIORITY STRATEGY

\begin{tabular}{c|c|c|c|c|c|c|c}
\hline \multicolumn{2}{c|}{ nj } & dj & pj & pi & cpi & \multicolumn{2}{c}{ ni } \\
\hline 0 & 0 & 102 & 20 & 8 & 135 & 33 & 0 \\
\hline 0 & 49 & 79 & 18 & 10 & 82 & 36 & 0 \\
\hline 0 & 62 & 98 & 16 & 12 & 95 & 33 & 0 \\
\hline 0 & 52 & 85 & 14 & 14 & 73 & 21 & \\
\hline & & 80 & 13 & 16 & 90 & & \\
\hline
\end{tabular}

(Note: $\mathrm{nj}$ is the remaining of buyer $\mathrm{j}$, ni is the remaining of seller i)

Calculate the buyer's total profit $\mathrm{Pb}$, the seller's total profit Ps, and the market distribution total profit Ptotal:

$p_{b}=\sum_{j \in I_{b}, i \in I_{s}} q_{i j}\left(v_{j}-p_{i j}\right)=1226$

$$
\begin{aligned}
& p_{s}=\sum_{i \in I_{s}, j \in I_{b}} q_{i j}\left(p_{i j}-s l_{i}\right)=3998.8 \\
& p=p_{b}+p_{s}=1226+3998.8=5224.8
\end{aligned}
$$

Strategy two: The trading process and result of multistage market trading mechanism is shown in the following.

TABLE IV THE FIRST ROUND TRADING PROCESS

\begin{tabular}{c|c|c|c|c|c|c|c|c}
\hline $\mathrm{n} 1 \mathrm{j}$ & $\mathrm{dj}$ & $\mathrm{pj}$ & \multicolumn{3}{|c|}{$\mathrm{Wij}$} & $\max (\mathrm{Wij})$ \\
\hline 12 & 102 & 20 & 1428 & 1230 & 1520 & 1241 & 1620 & 1620 \\
\hline & 79 & 18 & 1027 & 1106 & 1185 & 1168 & 1343 & 1185 \\
\hline & 98 & 16 & 1176 & 1066 & 1330 & 1095 & 1440 & 1176 \\
\hline 12 & 85 & 14 & 935 & 984 & 1105 & 1022 & 1275 & 1022 \\
\hline & 80 & 13 & 840 & 920 & 1000 & 985.5 & 1160 & 920 \\
\hline & & & 8 & 10 & 12 & 14 & 16 & $\mathrm{pi}$ \\
\hline & & & 135 & 82 & 95 & 73 & 90 & $\mathrm{cpi}$ \\
\hline & & & 37 & 2 & 16 & & & $\mathrm{n} 1 \mathrm{i}$ \\
\hline
\end{tabular}

(Note: $\mathrm{n} 1 \mathrm{j}$ is the remaining of buyer $\mathrm{j}$ in the first round trading, $\mathrm{n} 1 \mathrm{i}$ is the remaining of seller $\mathrm{i}$ in the first round trading).

\section{TABLE V SECOND ROUND TRADING PROCESS}

\begin{tabular}{c|c|c|c|c|c|c}
\hline $\mathrm{n} 2 \mathrm{j}$ & $\mathrm{dj}$ & $\mathrm{pj}$ & $\mathrm{pi}$ & $\mathrm{cpi}$ & \multicolumn{2}{|c}{$\mathrm{n} 2 \mathrm{i}$} \\
\hline 0 & 12 & 20 & 8 & 37 & 25 & 13 \\
\hline 0 & 12 & 14 & 10 & 2 & & \\
\hline & & & 12 & 16 & & \\
\hline
\end{tabular}

(Note: $n 2 \mathrm{j}$ is the remaining of buyer $\mathrm{j}$ in the second round trading, $n 2 \mathrm{i}$ is the remaining of seller $\mathrm{i}$ in the second round trading).
Calculate the buyer's total profit $\mathrm{Pb}$, the seller's total profit Ps, and the market distribution total profit Ptotal:

$$
\begin{aligned}
& p_{b}=\sum_{j \in I_{b}, i \in I_{s}} q_{i j}\left(v_{j}-p_{i j}\right)=4852 \\
& p_{s}=\sum_{i \in I_{s}, j \in I_{b}} q_{i j}\left(p_{i j}-s l_{i}\right)=4975.74
\end{aligned}
$$




$$
p=p_{b}+p_{s}=4852+4975.74=9827.74
$$

Comparing the calculation results of the two trading strategies, we can see that the multi-stage market trading strategy can obtain more market total profits than the price priority strategy, therefore the strategy of the multi-stage market trading can allocate resources more effectively.

\section{THE EXPERIMENTAL RESULTS}

In order to further verify that the multi-stage market trading strategy can obtain a greater market total profit than the price priority strategy for resource allocation, the two transaction strategies are evaluated in five different network resource allocation systems, each systems consists of 20 sellers and 20 buyers. And it is according to the total market profit of two trading strategies to determine which strategy is more effective.

The agent settings: the range of selling fixed start cost fi is $(20,50)$, the range of unit cost ui is $(2,4)$, the range of provide capacity cpi is $(50,150)$, and the seller's asking price cannot exceed 22. The buyer's valuation vj ranges from 20 to 30 , the buyer's demand dj ranges from 60 to 120, and the buyer's bid cannot be less than 10 . Under the constraints that the buyer's bid must not be lower than the seller's asking price, after the transaction matching of system 1 to system 3 terminating, both the buyers' demand and the sellers' resource supply amount have remained; after the transaction matching of system 4 and system 5 terminating, there is no surplus in the buyers' demand but in the sellers' supply of resources. The experimental results are shown as following chart.

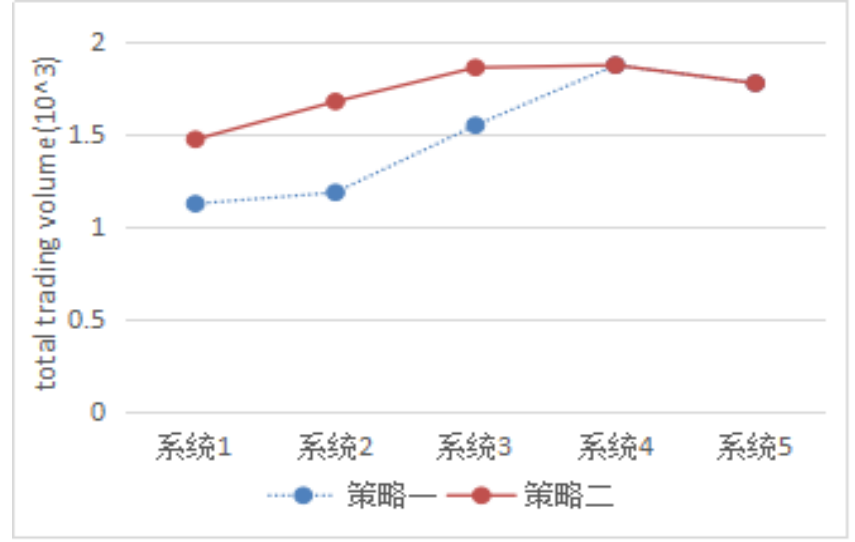

Fig. 1 Total trading volume of two Strategies

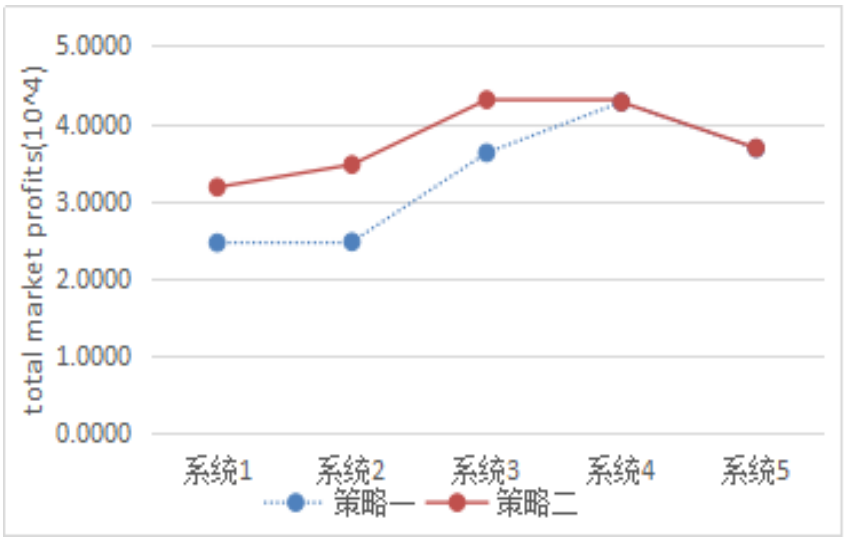

Fig. 2 Total market profits of two Strategies

As charts above showing that in the same system, if the buyer has the initiative, the transaction volume of the multistage market trading strategy are more than or equal to the price priority strategy. When the transaction volume of the multi-stage market trading strategy are more than the price priority strategy, the actual total market profit of the multistage market trading strategy are greater than the price priority strategy. When the transaction volume of them are same, the actual total market profits of them are approximately same. Therefore, in the same system, the multi-stage market trading strategy is more effective than the price priority trading strategy for resource allocation, and the multi-stage market trading strategy obtain greater market profit, only when the transaction volume of them are different.

\section{CONCLUSION}

Mainly based on the framework of the CDA market mechanism to solve the problem of large-scale network resource allocation. Under the premise that a buyer can enter into a transaction with multiple sellers and a seller can also conclude transactions with multiple buyers, the paper put forward the multi-stage market trading strategy and the price priority strategy, then calculated the actual total market profit of the two trading strategy by setting up calculation model. Experimental data shown that the multi-stage market trading strategy is more effective for the distribution of network resources.

\section{ACKNOWLEDGMENT}

This work was supported by the National Natural Science Foundation of China (71501147). Cultivation plan project for young scholar at Wuhan University of Science and Technology (250089) and Center of Service Science and Engineering at Wuhan University of Science and Technology (CSSE2017GB03). 


\section{REFERENCES}

[1] Wenjie Zhan, Yantao Bai. Research on Bidirectional Bidding Strategy Based on Dynamic Hurwicz Criterion. Journal of Management, 2014, 11(3):416-420. (In Chinese)

[2] Lingjuan Li, Huanhui Xie. Grid resource management model based on continuous double auction. Journal of Xi'an University of Posts and Telecommunications. 2008, 13(5). (In Chinese)

[3] Shengfeng Chen, Chengjian Wei. Rational strategy under the environment of limited resources and continuous two-way auction. Computer Engineering and Applications, 2011, 47(15):241-244. (In Chinese)

[4] Phelp S, Parsons S. Applying programming to economic mechaanism des- ign [C]. Proceeding of the second International Joint Conference on Autonomous Agents and Multiagent Systerm, 2003:1096-1097.

[5] Dash R K.Distributed mechannisms for multi-agent systems: Analysis and Design [D]. Southampton: University of Southamption, 2006.

[6] Yajuan Wang, Xianjia Wang. An incentive-compatible power generation Trading Bilateral Auction Mechanism. Power System Automation, 2009, 33(22):25-28. (In Chinese)

[7] Gode D K, Sunder S. Allocative efficiency of markets with zero intelligence traders: Market as a partial substitute for individual rationality [J]. Journal of Political Economy, 1993, 101(1):119-137.

[8] Cliff D, Bruten J. Minimal-intelligence agents for bargaining behavi- ors in market-based environments, Technical Report HPL [R]. Hewlett Packard Labs, 1997.

[9] Gjerstad S, Dickhaut J. Price formation in double auction[J].Games and Economic Behavior,1998,22(1):1-29. 